Research Article

Moosa Gabeleh* and Hans-Peter A. Künzi

\title{
Equivalence of the existence of best proximity points and best proximity pairs for cyclic and noncyclic nonexpansive mappings
}

https://doi.org/10.1515/dema-2020-0005

received July 4, 2019; accepted January 26, 2020

Abstract: In this study, at first we prove that the existence of best proximity points for cyclic nonexpansive mappings is equivalent to the existence of best proximity pairs for noncyclic nonexpansive mappings in the setting of strictly convex Banach spaces by using the projection operator. In this way, we conclude that the main result of the paper [Proximal normal structure and nonexpansive mappings, Studia Math. 171 (2005), 283-293] immediately follows. We then discuss the convergence of best proximity pairs for noncyclic contractions by applying the convergence of iterative sequences for cyclic contractions and show that the convergence method of a recent paper [Convergence of Picard's iteration using projection algorithm for noncyclic contractions, Indag. Math. 30 (2019), no. 1, 227-239] is obtained exactly from Picard's iteration sequence.

Keywords: best proximity (point) pair, uniformly convex Banach space, noncyclic (cyclic) contraction

MSC 2010: 47H09, 46B20

\section{Introduction}

Throughout this study, $(A, B)$ is a pair of nonempty and disjoint subsets of a normed linear space $X$. A mapping $T: A \cup B \rightarrow A \cup B$ is said to be cyclic if $T(A) \subseteq B$ and $T(B) \subseteq A$. Also, $T$ is called a noncyclic mapping $T(A) \subseteq A$ and $T(B) \subseteq B$.

Definition 1.1. A point $(p, q) \in A \times B$ is said to be a best proximity pair for the noncyclic mapping $T: A \cup B \rightarrow A \cup B$ provided that

$$
p=T p, \quad q=T q \quad \text { and } d(p, q)=\operatorname{dist}(A, B):=\inf \{d(x, y):(x, y) \in A \times B\} .
$$

Also, if $T: A \cup B \rightarrow A \cup B$ is a cyclic mapping, then a point $x^{\star} \in A \cup B$ is called a best proximity point for $T$ provided that

$$
\left\|x^{\star}-T x^{\star}\right\|=\operatorname{dist}(A, B) .
$$

Some existence results of best proximity points (pairs) can be found in [1-5].

Definition 1.2. $T: A \cup B \rightarrow A \cup B$ is called a cyclic (noncyclic) nonexpansive mapping, if $T$ is cyclic (noncyclic) and

$$
\|T x-T y\| \leq\|x-y\|, \quad \forall(x, y) \in A \times B .
$$

\footnotetext{
* Corresponding author: Moosa Gabeleh, Department of Mathematics, Ayatollah Boroujerdi University, Boroujerd, Iran, e-mail: Gabeleh@abru.ac.ir, gab.moo@gmail.com

Hans-Peter A. Künzi: Department of Mathematics and Applied Mathematics, University of Cape Town, Rondebosch 7701, South Africa, e-mail: hans-peter.kunzi@uct.ac.za
} 
Also, $T$ is said to be a cyclic (noncyclic) contraction provided that $T$ is cyclic (noncyclic) and there exists $\alpha \in(0,1)$ for which

$$
d(T x, T y) \leq \alpha d(x, y)+(1-\alpha) \operatorname{dist}(A, B),
$$

for all $(x, y) \in A \times B$.

It is clear that the class of cyclic (noncyclic) nonexpansive mappings contains the class of cyclic (noncyclic) contractions as a subclass.

In order to state the existence and convergence results of best proximity points (pairs), we need to recall the following concepts and notations.

Definition 1.3. A Banach space $X$ is said to be

(i) uniformly convex if there exists a strictly increasing function $\delta:[0,2] \rightarrow[0,1]$ such that the following implication holds for all $x, y, p \in X, R>0$ and $r \in[0,2 R]$ :

$$
\left\{\begin{array}{l}
\|x-p\| \leq R, \\
\|y-p\| \leq R, \\
\|x-y\| \geq r
\end{array} \Rightarrow\left\|\frac{x+y}{2}-p\right\| \leq\left(1-\delta\left(\frac{r}{R}\right)\right) R ;\right.
$$

(ii) strictly convex if the following implication holds for all $x, y, p \in X$ and $R>0$ :

$$
\left\{\begin{array}{l}
\|x-p\| \leq R, \\
\|y-p\| \leq R, \\
x \neq y
\end{array} \Rightarrow\left\|\frac{x+y}{2}-p\right\|<R .\right.
$$

The proximal pair of the pair $(A, B)$ is denoted by $\left(A_{0}, B_{0}\right)$ and given by

$$
\begin{aligned}
& A_{0}=\left\{x \in A:\left\|x-y^{\prime}\right\|=\operatorname{dist}(A, B) \text { for some } y^{\prime} \in B\right\}, \\
& B_{0}=\left\{y \in B:\left\|x^{\prime}-y\right\|=\operatorname{dist}(A, B) \text { for some } x^{\prime} \in A\right\} .
\end{aligned}
$$

The pair $(A, B)$ is said to be a proximinal pair if $A=A_{0}$ and $B=B_{0}$.

We shall also adopt the notation

$$
\begin{aligned}
\delta_{x}(A) & =\sup \{d(x, y): y \in A\} \text { for all } x \in X, \\
\delta(A, B) & =\sup \{d(x, y): x \in A, y \in B\}, \\
\operatorname{diam}(A) & =\delta(A, A)
\end{aligned}
$$

Definition 1.4. [6] A convex pair $\left(K_{1}, K_{2}\right)$ in a Banach space $X$ is said to have a proximal normal structure (PNS) if for any bounded, closed, convex and proximinal pair $\left(H_{1}, H_{2}\right) \subseteq\left(K_{1}, K_{2}\right)$ for which $\operatorname{dist}\left(H_{1}, H_{2}\right)=$ $\operatorname{dist}\left(K_{1}, K_{2}\right)$ and $\delta\left(H_{1}, H_{2}\right)>\operatorname{dist}\left(H_{1}, H_{2}\right)$, there exists $\left(x_{1}, x_{2}\right) \in H_{1} \times H_{2}$ such that

$$
\max \left\{\delta_{x_{1}}\left(H_{2}\right), \delta_{x_{2}}\left(H_{1}\right)\right\}<\delta\left(H_{1}, H_{2}\right) .
$$

It was announced in [6] that every nonempty, bounded, closed and convex pair in a uniformly convex Banach space $X$ has PNS.

Here, we state the following two existence results which are the main conclusions of [6].

Theorem 1.5. [6, Theorem 2.1] Let $(A, B)$ be a nonempty, weakly compact and convex pair in a Banach space $X$ and suppose $(A, B)$ has PNS. Let $T: A \cup B \rightarrow A \cup B$ be a cyclic nonexpansive mapping. Then, $T$ has a best proximity point. 
Theorem 1.6. [6, Theorem 2.2] Let $(A, B)$ be a nonempty, weakly compact and convex pair in a strictly convex Banach space $X$ and suppose $(A, B)$ has PNS. Let $T: A \cup B \rightarrow A \cup B$ be a noncyclic nonexpansive mapping. Then, $T$ has a best proximity pair.

In 2006, the next existence, uniqueness and convergence result of a best proximity point for cyclic contractions was established.

Theorem 1.7. [7, Theorem 3.10] Let $(A, B)$ be a nonempty, closed and convex pair in a uniformly convex Banach space $X$ and let $T: A \cup B \rightarrow A \cup B$ be a cyclic contraction map. For $x_{0} \in A$, define $x_{n+1}:=T x_{n}$ for each $n \geq 0$. Then, there exists a unique $x^{\star} \in A$ such that $x_{2 n} \rightarrow x^{\star}$ and $\left\|x^{\star}-T x^{\star}\right\|=\operatorname{dist}(A, B)$.

Just recently, the noncyclic version of Theorem 1.7 was proved in [8]. Before stating that we recall the following requirements.

For a nonempty subset $A$ of $X$ a metric projection operator $\mathcal{P}_{A}: X \rightarrow 2^{A}$ is defined as

$$
\mathcal{P}_{A}(x):=\{y \in A:\|x-y\|=\operatorname{dist}(\{x\}, A)\},
$$

where $2^{A}$ denotes the set of all subsets of $A$. It is well known that if $A$ is a nonempty, closed and convex subset of a reflexive and strictly convex Banach space $X$, then the metric projection $\mathcal{P}_{A}$ is single valued from $X$ to $A$, that is, $\mathcal{P}_{A}: X \rightarrow A$ is a mapping with $\left\|x-\mathcal{P}_{A}(x)\right\|=\operatorname{dist}(\{x\}, A)$ for any $x \in X$.

Proposition 1.8. $[8,9]$ Let $(A, B)$ be a nonempty, bounded, closed and convex pair in a reflexive and strictly convex Banach space X. Define $\mathcal{P}: A_{0} \cup B_{0} \rightarrow A_{0} \cup B_{0}$ as

$$
\mathcal{P}(x)= \begin{cases}\mathcal{P}_{A_{0}}(x) & \text { if } x \in B_{0}, \\ \mathcal{P}_{B_{0}}(x) & \text { if } x \in A_{0} .\end{cases}
$$

Then, the following statements hold.

(1) $\mathcal{P}$ is cyclic on $A_{0} \cup B_{0}$ and $\left\|x-\mathcal{P}_{X}\right\|=\operatorname{dist}(A, B)$ for any $x \in A_{0} \cup B_{0}$,

(2) $\mathcal{P}$ is an isometry, that is, $\left\|\mathcal{P}_{x}-\mathcal{P} y\right\|=\|x-y\|$ for all $(x, y) \in A_{0} \times B_{0}$,

(3) $\mathcal{P}$ is affine,

(4) $\left.\mathcal{P}^{2}\right|_{A_{0}}=i_{A_{0}}$ and $\left.\mathcal{P}^{2}\right|_{B_{0}}=i_{B_{0}}$, where $i_{E}$ is the identity mapping on the subset $E$ of $X$.

Moreover, if $X$ is a uniformly convex Banach space, then

(5) $\left.\mathcal{P}\right|_{A_{0}}$ and $\left.\mathcal{P}\right|_{B_{0}}$ are continuous.

We are now ready to state a main result of [8].

Theorem 1.9. [8, Theorem 3.2] Let $(A, B)$ be a nonempty, closed and convex pair in a uniformly convex Banach space $X$ and $T$ a noncyclic contraction mapping defined on $A \cup B$. Suppose $x_{0} \in A_{0}$ and define

$$
\left\{\begin{array}{c}
x_{n}=T^{n} x_{0}, \\
y_{n}=\mathcal{P} x_{n},
\end{array}\right.
$$

for all $n \in \mathbb{N}$, where $\mathcal{P}$ is the projection operator defined in (1). Then, the sequence $\left\{\left(x_{n}, y_{n}\right)\right\} \subseteq A_{0} \times B_{0}$ converges to a best proximity pair of the mapping $T$.

The main purpose of this study is to show that the existence of best proximity points for cyclic nonexpansive mappings is equivalent to the existence of best proximity pairs for noncyclic nonexpansive mappings in the setting of strictly convex Banach spaces. Then, we conclude that Theorem 1.6 is a straightforward consequence of Theorem 1.5. We also obtain a stronger version of Theorem 1.9 by using Theorem 1.7. 


\section{Main results}

We begin our main conclusions with the following theorem.

Theorem 2.1. Let $(A, B)$ be a nonempty, weakly compact and convex pair in a strictly convex Banach space $X$. Then, every cyclic nonexpansive mapping defined on $A \cup B$ has a best proximity point if and only if every noncyclic nonexpansive mapping defined on $A \cup B$ has a best proximity pair.

Proof. Assume that every cyclic nonexpansive mapping defined on $A \cup B$ has a best proximity point and $T: A \cup B \rightarrow A \cup B$ is a noncyclic nonexpansive mapping. If $x \in A_{0}$, then there exists a point $y \in B_{0}$ for which $\|x-y\|=\operatorname{dist}(A, B)$. By the fact that $T$ is a noncyclic nonexpansive mapping, we obtain $\|T x-T y\|=\operatorname{dist}$ $(A, B)$ and so $T x \in A_{0}$ which ensures that $T\left(A_{0}\right) \subseteq A_{0}$. Similarly, $T\left(B_{0}\right) \subseteq B_{0}$, that is, $T$ is noncyclic on $A_{0} \cup B_{0}$. Consider the projection operator $\mathcal{P}$ as in (1). It follows from the proof of Theorem 3.2 of [8] that $T$ and $\mathcal{P}$ commute on $A_{0} \cup B_{0}$. Since $\mathcal{P}$ is cyclic and $T$ is noncyclic on $A_{0} \cup B_{0}$, we obtain

$$
T \mathcal{P}\left(A_{0}\right) \subseteq T\left(B_{0}\right) \subseteq B_{0}, \quad T \mathcal{P}\left(B_{0}\right) \subseteq T\left(A_{0}\right) \subseteq A_{0} .
$$

Therefore, $T \mathcal{P}$ is cyclic on $A_{0} \cup B_{0}$. In view of the fact that $\mathcal{P}$ is an isometry,

$$
\|T \mathcal{P} x-T \mathcal{P} y\|=\|\mathcal{P} T x-\mathcal{P} T y\|=\|T x-T y\| \leq\|x-y\|,
$$

for all $(x, y) \in A_{0} \times B_{0}$, that is, $T \mathcal{P}$ is a cyclic nonexpansive mapping on $A_{0} \cup B_{0}$. Now by assumption, there exists a point $x^{\star} \in A_{0}$ such that $\left\|x^{\star}-T \mathcal{P}_{X^{\star}}\right\|=\operatorname{dist}(A, B)$. Moreover,

$$
\left\|T x^{\star}-T \mathcal{P}^{\star}\right\|=\left\|T \chi^{\star}-\mathcal{P} T \chi^{\star}\right\|=\operatorname{dist}(A, B) .
$$

Strict convexity of $X$ implies that $T x^{\star}=x^{\star}$. Furthermore,

$$
\mathcal{P} X^{\star}=\mathcal{P} T X^{\star}=T \mathcal{P} X^{\star}
$$

Hence, $\left(x^{\star}, \mathcal{P} x^{\star}\right)$ is a best proximity pair of the mapping $T$. Conversely, assume that any noncyclic nonexpansive mapping defined on $A_{0} \cup B_{0}$ has a best proximity pair and $S: A \cup B \rightarrow A \cup B$ is a cyclic nonexpansive mapping. Thus,

$$
S \mathcal{P}\left(A_{0}\right) \subseteq S\left(B_{0}\right) \subseteq A_{0}, \quad S \mathcal{P}\left(B_{0}\right) \subseteq S\left(A_{0}\right) \subseteq B_{0},
$$

that is, $S \mathcal{P}$ is noncyclic on $A_{0} \cup B_{0}$ and again since $\mathcal{P}$ is an isometry, we conclude that $S \mathcal{P}$ is a noncyclic nonexpansive mapping. Now by the assumption $S \mathcal{P}$ has a best proximity pair, called $(p, q) \in A_{0} \times B_{0}$. Thereby,

$$
S \mathscr{P} p=p, \quad S \mathscr{P} q=q, \quad\|p-q\|=\operatorname{dist}(A, B) .
$$

We have

$$
\|p-S p\|=\|S \mathcal{P} p-S p\| \leq\|\mathcal{P} p-p\|=\operatorname{dist}(A, B),
$$

and so $p$ is a best proximity point of $S$. Similarly, we can see that $q$ is a best proximity point of $S$ in $B_{0}$ and this completes the proof.

Corollary 2.2. Theorem 1.6 is a consequence of Theorem 1.5 (see the proof of Theorem 1.6 in [6]).

Here, we compare Theorem 1.7 with Theorem 1.9.

Theorem 2.3. Theorem 1.9 implies Theorem 1.7 when the initial point of the iterative sequence in Theorem 1.7 is chosen in $A_{0}$. 
Proof. Let $(A, B)$ be a nonempty, closed and convex pair in a uniformly convex Banach space $X$ and $S: A \cup B \rightarrow A \cup B$ be a cyclic contraction. From Proposition 3.3 of [7], the pair $\left(A_{0}, B_{0}\right)$ is nonempty. Consider the mapping $S \mathscr{P}$ on $A_{0} \cup B_{0}$. A proof which is similar to the one of Theorem 2.1 shows that the mapping $S \mathscr{P}$ is a noncyclic contraction on $A_{0} \cup B_{0}$. Thus, for any $x_{0} \in A_{0}$ if we define

$$
\left\{\begin{array}{l}
x_{n}=(S \mathcal{P})^{n} x_{0} \\
y_{n}=\mathcal{P}_{X_{n}}
\end{array}\right.
$$

then $\left\{\left(x_{n}, y_{n}\right)\right\}$ converges to a best proximity pair of the mapping $S \mathcal{P}$. Suppose $x_{n} \rightarrow p$. Continuity of the projection operator $\mathcal{P}$ implies that $y_{n} \rightarrow \mathcal{P} p$. Since $\mathcal{P}^{2}$ is identity on $A_{0}$ and $B_{0}$, respectively (Proposition 1.8),

$$
S \mathcal{P} p=p, \quad \mathcal{P} p=S \mathcal{P}(\mathcal{P} p)=S p,
$$

and

$$
\|p-S p\|=\|p-\mathcal{P} p\|=\operatorname{dist}(A, B) .
$$

Hence, $p \in A_{0}$ is a best proximity point for the mapping $S$. We also note that for any $n \in \mathbb{N}$

$$
x_{2 n}=(S \mathcal{P})^{2 n} x_{0}=S^{2 n} \mathcal{P}^{2 n} x_{0} \text { (since } S \text { and } \mathcal{P} \text { commute) }=S^{2 n} x_{0} \rightarrow p \text {. }
$$

Theorem 2.4. Theorem 1.7 implies Theorem 1.9 for an even subsequence of the iterative sequence defined in (2).

Proof. Let $(A, B)$ be a nonempty, closed and convex pair in a uniformly convex Banach space $X$ and $T: A \cup B \rightarrow A \cup B$ be a noncyclic contraction. From Proposition 3.4 of [10], the pair $\left(A_{0}, B_{0}\right)$ is nonempty. Consider the mapping $T \mathcal{P}$ on $A_{0} \cup B_{0}$. The mapping $T \mathcal{P}$ is a cyclic contraction on $A_{0} \cup B_{0}$. Thus, for all $x_{0} \in A_{0}$ if we define $x_{n}=(T \mathcal{P})^{n} x_{0}$, then the sequence $\left\{x_{2 n}\right\}$ converges to a best proximity point of $T \mathcal{P}$, say $x^{\star} \in A_{0}$. In view of the fact that $T \mathcal{P}$ is a cyclic nonexpansive mapping by Theorem 2.1, we conclude that $\left(x^{\star}, \mathcal{P} \chi^{\star}\right)$ is a best proximity pair for $T$. From Proposition 1.8 , since $\mathcal{P}^{2}$ is identity map on $A_{0}$, we must have

$$
x_{2 n}=(T \mathcal{P})^{2 n} x_{0}=T^{2 n} \mathcal{P}^{2 n} x_{0}=T^{2 n} x_{0} \rightarrow x^{\star} .
$$

Continuity of the projection operator on $A_{0}$ ensures that $y_{2 n}:=\mathcal{P}_{X_{2 n}} \rightarrow \mathcal{P} X^{\star}$ and so the sequence $\left\{\left(x_{2 n}, \mathcal{P} x_{2 n}\right)\right\}$ converges to a best proximity pair of $T$. Notice that for any $n \in \mathbb{N}$ we have

$$
x_{2 n+1}=(T \mathcal{P})^{2 n+1} x_{0}=T^{2 n+1} \mathcal{P}^{2 n+1} x_{0}=T^{2 n+1} \mathcal{P} x_{0} \in B_{0} .
$$

Corollary 2.5. The convergence results of Theorem 1.7 and Theorem 1.9 are independent. That is, the convergence result of Theorem 1.7 cannot be implied by the convergence result of Theorem 1.9 and vice versa.

Example 2.1. Consider the Banach space $X=\mathbb{R}^{2}$ and let

$$
A=\{(x, y): 0 \leq x \leq 1,0 \leq y \leq x\}, \quad B=[3,4] \times[0,1] .
$$

Then,

$$
A_{0}=\{(1, y): 0 \leq y \leq 1\}, \quad B_{0}=\{(3, v): 0 \leq v \leq 1\} .
$$

It is worth noticing that the projection operator $\mathcal{P}: A_{0} \cup B_{0} \rightarrow A_{0} \cup B_{0}$ is defined by

$$
\mathcal{P}(1, y)=(3, y), \quad \mathcal{P}(3, v)=(1, v),
$$


where $y, v \in[0,1]$. For $((x, y),(u, v)) \in A \times B$, define the mapping $T: A \cup B \rightarrow A \cup B$ by

$$
T(x, y)=\left(\frac{x+1}{2}, y\right), \quad T(u, v)=\left(\frac{u+3}{2}, v\right) .
$$

Obviously, $T$ is noncyclic on $A \cup B$. Also for any $((x, y),(u, v)) \in A \times B$, we can see easily,

$$
\|T(x, y)-T(u, v)\|=\sqrt{\left|\frac{x+1}{2}-\frac{u+3}{2}\right|^{2}+|y-v|^{2}} \leq \sqrt{|x-u|^{2}+|y-v|^{2}},
$$

that is, $T$ is noncyclic nonexpansive. Moreover,

$$
T \mathcal{P}\left(A_{0}\right)=T\left(B_{0}\right)=B_{0}, \quad T \mathcal{P}\left(B_{0}\right)=T\left(A_{0}\right)=A_{0},
$$

which ensures that the mapping $T \mathcal{P}: A_{0} \cup B_{0} \rightarrow A_{0} \cup B_{0}$ is cyclic and

$$
\|T \mathcal{P}(1, y)-T \mathcal{P}(3, v)\|=\|T(3, y)-T(1, v)\|=\|(3, y)-(1, v)\|,
$$

for all $0 \leq y, v \leq 1$ which implies that the mapping $T \mathcal{P}$ is cyclic nonexpansive. Now for any $(1, y) \in A_{0}$ we have

$$
\|(1, y)-T \mathscr{P}(1, y)\|=\|(1, y)-T(3, y)\|=\|(1, y)-(3, y)\|=2=\operatorname{dist}(A, B),
$$

that is, $(1, y) \in A_{0}$ is a best proximity point for the mapping $T^{\mathcal{P}}$. We also note that

$$
((1, y), \mathcal{P}(1, y))=((1, y),(3, y)) \in A_{0} \times B_{0}
$$

is a best proximity pair of $T$.

Acknowledgments: Moosa Gabeleh would like to thank Ayatollah Boroujerdi University for financial support. Hans-Peter A. Künzi would also like to thank the National Research Foundation of South Africa for partial financial support (Grant Number: 118517).

\section{References}

[1] M. Al-Thagafi and N. Shahzad, Convergence and existence results for best proximity points, Nonlinear Anal. 70 (2009), 3665-3671.

[2] M. De la Sen, Linking contractive self-mappings and cyclic Meir-Keeler contractions with Kannan self-mappings, Fixed Point Theory Appl. 2010 (2010), DOI: 10.1155/2010/572057.

[3] M. De la Sen and R. P. Agarwal, Some fixed point-type results for a class of extended cyclic self-mappings with a more general contractive condition, Fixed Point Theory Appl. 2011 (2011), DOI: 10.1186/1687-1812-2011-59.

[4] S. Karpagam and S. Agrawal, Best proximity point theorems for p-cyclic Meir-Keeler contractions, Fixed Point Theory Appl. 2009 (2009), DOI: 10.1155/2009/197308.

[5] M. Petric and B. Zlatanov, Best proximity points for p-cyclic summing iterated contractions, Filomat 32 (2018), $3275-3287$.

[6] A. A. Eldred, W. A. Kirk, and P. Veeramani, Proximal normal structure and relatively nonexpansive mappings, Studia Math. 171 (2005), 283-293.

[7] A. A. Eldred and P. Veeramani, Existence and convergence of best proximity points, J. Math. Anal. Appl. 323 (2006), 1001-1006.

[8] M. Gabeleh, Convergence of Picard's iteration using projection algorithm for noncyclic contractions, Indag. Math. 30 (2019), 227-239.

[9] M. Gabeleh, Common best proximity pairs in strictly convex Banach spaces, Georgian Math. J. 24 (2017), 363-372.

[10] A. Fernández-León and M. Gabeleh, Best proximity pair theorems for noncyclic mappings in Banach and metric spaces, Fixed Point Theory 17 (2016), no. 1, 63-84. 